International Mathematical Forum, 2, 2007, no. 24, 1211 - 1214

\title{
Real Divisors of a Projective Variety Containing a Given Scheme
}

\author{
E. Ballico ${ }^{1}$ \\ Dept. of Mathematics \\ University of Trento \\ 38050 Povo (TN), Italy \\ ballico@science.unitn.it
}

\begin{abstract}
Let $Z \subset X \subset \mathbf{P}^{n}$ be real projective schemes with $X$ integral and $Z$ of codimension at least 2 in $X$. Here we find a real hypersurface of $X$ containing $Z$, with bounded degree and with other properties.
\end{abstract}

Mathematics Subject Classification: 14P05; 14P20

Keywords: real algebraic variety; real divisor; degree of a hypersurface

\section{INTRODUCTION}

Let $Z \subset X \subset \mathbf{P}^{n}$ be real projective schemes with $X$ integral and $Z$ of codimension at least 2 in $X$. Here (under suitable assumptions) we find a real hypersurface $X(f)$ of $X$ containing $Z$, with bounded degree and with other properties. In Theorem 1 we consider the case $X(f)(\mathcal{R})=Z(\mathcal{R})$. In Theorem 2 we find $X(f)$ whose real locus intersects many connected components of $X_{\text {reg }}(\mathcal{R})$ for the semialgebraic topology.

Lemma 1. Let $X \subset \mathbf{P}^{n}$ be an integral projective variety defined over an algebraically closed field $\mathbb{K}$ with char $(\mathbb{K})=0$ and $Z \subset X$ a closed subscheme with codimension $c \geq 2$. Let $\mu$ denote the minimal integer $t$ such that $Z_{\text {red }}$ is the set-theoretic base locus of $H^{0}\left(\mathbf{P}^{n}, \mathcal{I}_{Z}(t)\right)$. Fix a finite $S \subseteq Z_{\text {reg }} \cap X_{\text {reg }}$ (we allow the case $S=\emptyset)$. Assume also that the scheme-theoretic base locus of $H^{0}\left(\mathbf{P}^{n}, \mathcal{I}_{Z}(t)\right)$ is equal to $Z$ in a neighborhood of $S$. Fix an integer e such that $1 \leq e \leq c-1$, an integer $d \geq \mu$ and a general $e$-dimensional linear subspace of $H^{0}\left(\mathbf{P}^{n}, \mathcal{I}_{Z}(d)\right)$. Let $A(V, X)$ the scheme-theoretic intersection of $X$ with the codimension $e$ and degree $d^{e}$ complete intersection $A(V):=\{f=0\}_{f \in V \backslash\{0\}}$.

(i) $A(V, X)$ has dimension $\operatorname{dim}(X)-e$ and it is smooth at each point of $X_{\text {reg }} \backslash Z$ and at each point of $S$.

\footnotetext{
${ }^{1}$ The author was partially supported by MIUR and GNSAGA of INdAM (Italy).
} 
(ii) If $d>\mu$, then $A(V, X)$ is integral;

(iii) Assume $d=\mu ; A(V, X)$ is integral if for a general $P \in X$ the base locus of $H^{0}\left(\mathbf{P}^{n}, \mathcal{I}_{Z \cup\{P\}}(d)\right)$ has dimension at most $\operatorname{dim}(X)-e-1$.

Proof. Part (i) outside $S$ follows from Bertini's theorem ([3], part 2) of Th. 6.3). Since $S$ is finite, it is sufficient to notice that for general $V$ and any fixed $P \in Z, T_{P} V$ is transversal to $T_{P} X$ by our assumption on $S$. Part (iii) for an arbitrary $d \geq \mu$ is true by another theorem of Bertini ([3], part 4) of Th. 6.3). It is easy to check that if $d>\mu$, then the condition in (iii) is satisfied (use reducible hypersurfaces, union of hypersurfaces of degree $\mu$ containg $Z$ and arbitrary degree $d-\mu$ hypersurfaces. Part (ii) for $S=\emptyset$ was also checked in [1], proof of Th. 1.2).

Quite often, but not always (e.g. if $X=\mathbf{P}^{n}$ and $Z$ is a point) in part (iii) the "only if" assertion holds.

Remark 1. Take $X, Z, S, d$ as in Lemma 1. Assume that $X, Z, S$ are defined over a real closed field $\mathcal{R}$ and that $\mathbb{K}$ is the algebraic closure of $\mathbb{R}$. Notice that $H^{0}\left(\mathbf{P}^{n}, \mathcal{I}_{Z}(d)\right)=H^{0}\left(\mathbf{P}_{\mathcal{R}}^{n}, \mathcal{I}_{Z}(d)\right) \otimes_{\mathcal{R}} \otimes \mathbb{K}$, because any extension of fields is flat and cohomology commutes with base change ([2], Prop. III.9.3). Since $H^{0}\left(\mathbf{P}_{\mathcal{R}}^{n}, \mathcal{I}_{Z}(t)\right)$ is Zariski dense in $H^{0}\left(\mathbf{P}^{n}, \mathcal{I}_{Z}(d)\right)$, and smoothness is an open condition, we may take $V$ as in the statement of Lemma 1 with $V$ defined over $\mathcal{R}$ and (in parts (ii) and (iii)) with $A(V, X)$ geometrically irreducible.

Theorem 1. Let $\mathcal{R}$ be a real closed field and $\mathbb{K}$ its algebraic closure. Let $X \subset \mathbf{P}^{n}$ (resp. $Z \subset X$ ) a geometrically integral (resp. a closed subscheme of codimension at least 2 ) defined over $\mathbb{R}$. Let $\mu$ denote the minimal integer $t$ such that $Z_{\text {red }}$ is the set-theoretic base locus of $H^{0}\left(\mathbf{P}^{n}, \mathcal{I}_{Z}(t)\right)$. Fix an even integer $k \geq 2 \mu$. If $k=2 \mu$ assume that for a general $P \in X(\mathbb{K})$ the base locus of $H^{0}\left(\mathbf{P}^{n}, \mathcal{I}_{Z \cup\{P\}}(\mu)\right)$ in $X(\mathbb{K})$ has dimension at most $\operatorname{dim}(X)-2$. Then there $i s$ $f \in H^{0}\left(\mathbf{P}_{\mathcal{R}}^{n}, \mathcal{I}_{Z}(k)\right)$ such that $X(f):=X \cap\{f=0\}$ is a geometrically integral hypersurface of $\left.X,\left(X(\mathbb{K})_{\text {reg }} \cap X(f)\right) \backslash Z(\mathbb{K})\right) \subseteq X(f)(\mathbb{K})_{\text {reg }}$ and $X(f)(\mathcal{R})=$ $Z(\mathcal{R})$.

Proof. Set $d:=k / 2$. Take a basis $g_{1}, \ldots g_{s}$ of $H^{0}\left(\mathbf{P}_{\mathcal{R}}^{n}, \mathcal{I}_{Z}(k)\right)$. Hence $Z_{\text {red }}=$ $\left\{g_{1}=\cdots=g_{s}=0\right\}$. Apply the proofs of Lemma 1 and of Remark 1 for $e=1$ to the $\mathbb{K}$-vector space spanned by $g_{1}^{2}, \ldots, g_{s}^{2}$. Since $\left(\mathcal{R}_{>0}^{n}\right.$ is Zariski dense in $\mathbb{K}^{n}$ we obtain the existence of $c_{i} \in \mathcal{R}, 1 \leq i \leq s, c_{i}>0$ for all $i$, such that, setting $f=\sum_{i=1}^{s} c_{i} g_{i}^{2}, X(f):=X \cap\{f=0\}$ is a geometrically integral hypersurface of $X$ and $\left.\left(X(\mathbb{K})_{\text {reg }} \cap X(f)\right) \backslash Z(\mathbb{K})\right) \subseteq X(f)(\mathbb{K})_{\text {reg }}$. Since $c_{i}>0$ for all $i$ and each $g_{i}$ is real, we have $\{f=0\}(\mathcal{R})=Z(\mathcal{R})$. Hence $X(f)(\mathcal{R})=Z(\mathcal{R})$.

Notation 1. Let $X \subset \mathbf{P}^{n}$ be an integral projective variety defined over an algebraically closed field with $\operatorname{char}(\mathbb{K})=0$ and $Z \subset X$ a closed subscheme with codimension $c \geq 2$. For every integer $d$ let $W(X, d)$ denote the image of the restriction map $H^{0}\left(\mathbf{P}^{n}, \mathcal{O}_{\mathbf{P}^{n}}(d)\right) \rightarrow H^{0}\left(X, \mathcal{O}_{X}(d)\right)$. Set $W(X, d, Z):=W(X, d) \cap$ $H^{0}\left(X, \mathcal{I}_{Z, X}(d)\right), w(X, d):=\operatorname{dim}(W(X, d)$ and $w(X, d, Z):=\operatorname{dim}(W(X, d, Z))$. 
Let $\alpha(X, d)$ (resp. $\alpha(X, d, Z))$ denote the maximal integer $t \geq 0$ such that for a general $S \subset X$ the base locus of $W(X, d) \cap H^{0}\left(X, \mathcal{I}_{S}(d)\right)(\operatorname{resp} . W(X, d, Z) \cap$ $\left.H^{0}\left(X, \mathcal{I}_{S}(d)\right)\right)$ does not contain a codimension 1 subscheme of $X$ with the convention $\alpha(X, d)=-\infty$ (resp. $\alpha(X, d, Z)=-\infty)$ if no such integer $t \geq 0$ exists.

Remark 2. Let $\mathcal{R}$ be a real closed field with algebraic closure $\mathbb{K}$. In the setup of Notation 1 assume that $X, Z$ and the embedding $X \subset \mathbf{P}^{n}$ are defined over $\mathcal{R}$. Assume $X_{\text {reg }}(\mathcal{R}) \neq \emptyset$. The last assumption implies that $X_{\text {reg }}(\mathcal{R})$ is Zariski dense in $X(\mathbb{K})$. Hence if $\alpha(X, d)$ (resp. $\alpha(X, d, Z))$ is finite, then $\alpha(X, d)$ (resp. $\alpha(X, d, Z))$ is the maximal integer $t \geq 0$ such that there is $S \subset X_{\text {reg }}$ such that $\sharp(S)=t, \operatorname{dim}\left(W(X, d) \cap H^{0}\left(X, \mathcal{I}_{S}(d)\right)\right)=w(X, d)-t$ (resp. $\left.\operatorname{dim}\left(W(X, d, Z) \cap H^{0}\left(X, \mathcal{I}_{S}(d)\right)\right)=w(X, d, Z)-t\right)$ and the base locus of $W(X, d, Z) \cap H^{0}\left(X, \mathcal{I}_{S}(d)\right)$ (resp. $\left.W(X, d, Z) \cap H^{0}\left(X, \mathcal{I}_{S}(d)\right)\right)$ does not contain a codimension 1 subscheme of $X$. Let $T_{1}, \ldots, T_{s}$ be the connected components of $X_{\text {reg }}(\mathcal{R})$ in the semialgebraic topology. If $\mathcal{R}=\mathbb{R}$, then $T_{1}, \ldots, T_{s}$ are the connected components of $X_{\text {reg }}(\mathbb{R})$ in the euclidean topology. Fix integers $t_{i} \geq$ $0,1 \leq i \leq s$, such that $t_{1}+\cdots+t_{s}=t$. Since each $T_{i}$ is Zariski dense in $X(\mathbb{K})$, we may find $S$ as above and such that $\sharp\left(S \cap T_{i}\right)=t_{i}$ for all $i$.

Lemma 2. Let $X \subset \mathbf{P}^{n}$ be an integral projective variety defined over an algebraically closed field $\mathbb{K}$ with $\operatorname{char}(\mathbb{K})=0$ and $Z \subset X$ a closed subscheme with codimension $c \geq 2$. Fix an integer $d>0$ such that $h^{i}\left(\mathbf{P}^{n}, \mathcal{I}_{Z}(d-i)\right)=0$ for all $i>0$. Then $\alpha(X, d+1, Z) \geq w(X, d-1, Z)$.

Proof. Fix a general $S \subset X$ such that $\sharp(S)=w(X, d-1, Z)$. The generality of $S$ and the definition of the integer $w(X, d-1, Z)$ implies $h^{0}\left(\mathbf{P}^{n}, \mathcal{I}_{Z \cup S}(d-\right.$ $1))=h^{0}\left(\mathbf{P}^{n}, \mathcal{I}_{Z}(d-i)\right)-w(X, d-1, Z)$. Since $h^{1}\left(\mathbf{P}^{n}, \mathcal{I}_{Z}(d-1)\right)=0$, we get $h^{1}\left(\mathbf{P}^{n}, \mathcal{I}_{Z \cup S}(d-1)\right)=0$. Since $S$ is finite, we have $h^{i}\left(\mathbf{P}^{n}, \mathcal{I}_{Z \cup S}(d-i)\right)=$ $h^{i}\left(\mathbf{P}^{n}, \mathcal{I}_{Z}(d-i)\right)$ for all $i \geq 2$. Hence $h^{i}\left(\mathbf{P}^{n}, \mathcal{I}_{Z \cup S}(d-i)\right)=0$ for all $i>0$. By Castelnuovo-Mumford's lemma the homogeneous ideal of $S \cup Z$ in $\mathbf{P}^{n}$ is generated by forms of degree at most $d$. Apply part (ii) of Lemma 1 .

Remark 3. Take $X, Z, d$ as in Lemma 2 and a general $S \subset X$ such that $\sharp(S)=$ $w(X, d-1, Z)$. We saw in the proof of Lemma 2 that $h^{i}\left(\mathbf{P}^{n}, \mathcal{I}_{Z \cup S}(d-i)\right)=0$ for all $i>0$. By Castelnuovo-Mumford's lemma the homogeneous ideal of $S \cup Z$ in $\mathbf{P}^{n}$ is generated by forms of degree at most $d$. Thus we may apply part (i) of Lemma 1 to the set $Z^{\prime}:=Z \cup S$. As remarked in Lemma 2 we apply part (ii) of Lemma 1 to the set $Z^{\prime}$ and the integer $d^{\prime}:=d+1$ (and hence to all higher integers).

Lemma 3. Fix an integral projective variety $X \subset \mathbf{P}^{n}$ defined over an algebraically closed field $\mathbb{K}$ with char $(\mathbb{K})=0$, an integer $d>0$ and a vector space $V \subseteq H^{0}\left(X, \mathcal{O}_{X}(d)\right)$ such that $\operatorname{dim}(V) \geq 2$. Then fix a general $P \in X$ and set $V(-P):=\{f \in V: f(P)=0\}$. Let $\phi_{V}$ (resp. $\phi_{V(-P)}$ ) be the rational map on $X$ induced by the linear system $|V|$ (resp. $|V(-P)|)$. Hence 
$\operatorname{dim}(V(-P))=\operatorname{dim}(V)-1$. We have $\operatorname{dim}\left(\phi_{V(-P)}(X)=\operatorname{dim}\left(\phi_{V}(X)\right)\right.$ if and only if the rational map $\phi_{V}$ from $X$ into $\mathbf{P}\left(V^{*}\right)$ is not dominant.

Proof. The equality $\operatorname{dim}(V(-P))=\operatorname{dim}(V)-1$ is obvious, by the generality of $P$. Let $Y \subseteq \mathbf{P}\left(V^{*}\right)$ denote the closure of $\operatorname{Im}\left(\phi_{V}\right)$ in $\mathbf{P}\left(V^{*}\right)$. The linear projection of $Y$ from $Q \in Y$ maps $Y$ into a lower dimensional variety if and only $Y$ is a cone with vertex containing $Q$. Since $Y$ spans $\mathbf{P}\left(V^{*}\right)$, general $A \in Y$ is a vertex of $Y$ if and only if $Y=\mathbf{P}\left(V^{*}\right)$.

Remark 4. In the set-up of Lemma $3 \phi_{V}$ is not dominant if $\operatorname{dim}(V) \geq$ $\operatorname{dim}(X)+2$.

Theorem 2. Take the real closed field $\mathcal{R}$ as the base field. Let $X \subset \mathbf{P}^{n}$ be a geometrically integral variety and $Z \subset X$ a closed subscheme with codimension at least 2 in $X$. Let $T_{1}, \ldots, T_{s}$ denote the connected components of $X_{\text {reg }}(\mathcal{R})$ in the semialgebraic topology. Fix integers $d, k$ such that $h^{i}\left(\mathbf{P}^{n}, \mathcal{I}_{Z}(d-i)\right)=0$ for all $i>0$ and $d \geq k+1$. Let $w(X, k, Z)$ denote the dimension of the image of the restriction map $H^{0}\left(\mathbf{P}^{n}, \mathcal{I}_{Z}(k)\right) \rightarrow H^{0}\left(X, \mathcal{O}_{X}(d)\right)$. If $d=k+1$, then assume $w(X, k, Z) \geq \operatorname{dim}(X)+2$. Set $a:=\min \{s, w(X, k, Z)$. Then there exists a degree d hypersurface $Y \subset \mathbf{P}^{n}$ defined over $\mathcal{R}$ such that $Z \subset Y, X \cap Y$ is geometrically integral, $X_{\text {reg }} \cap Y \backslash Z$ is smooth and $Y$ intersects $T_{1}, \ldots, T_{a}$.

Proof. Apply Lemma 3 and Remarks 2 and 4.

Remark 5. If $X_{\text {reg }}(\mathcal{R}) \cap Z_{\text {reg }}(\mathcal{R}) \neq \emptyset$, then we may apply part (i) of Lemma 1 and get $X(f)$ as in the statement of Theorem 2 and smooth at a finite (prescribed in advance) set of $X_{\text {reg }}(\mathcal{R}) \cap Z_{\text {reg }}(\mathcal{R})$.

Remark 6. In the statements of Theorems 1 and 2 assume that $Z$ has codimension $c \geq 3$ in $X$. Fix an integer $e$ such that $2 \leq e \leq c-1$. Iterating the proof of Theorem 2 we may also find a complete intersection $Y$ of $e$ hypersurfaces of degree $d$ and with properties listed in the statement of Theorem 2 . This extension of Theorem 1 is even easier.

\section{REFERENCES}

[1] R. Ghiloni, Equations and complexity for the Dubois-Efroymson dimension theorem, preprint.

[2] R. Hartshorne, Alegraic Geometry, Springer, Berlin, 1977.

[3] J.-P. Jouanolou, Théorèmes de Bertini et applications, Progress in Math. 42, Birkhäuser, Basel, 1983.

\section{Received: October 7, 2006}

